\title{
Carbon Nanotube Coated High-Throughput Neurointerfaces in Assistive Environments
}

\author{
Mario I. Romero-Ortega \\ Department of Biomedical \\ Engineering \\ University of Texas at Arlington \\ Arlington, TX, USA \\ +1-817-272-5018 \\ mromero@uta.edu
}

\author{
Ali R. Butt \\ Department of Computer Science \\ Virginia Polytechnic Institute and \\ State University \\ Blacksburg, VA, USA \\ +1-540-231-0489 \\ butta@cs.vt.edu
}

\author{
Samir M. Iqbal \\ Department of Electrical Engineering \\ Nanotechnology Research and \\ Teaching Facility \\ University of Texas at Arlington \\ Arlington, TX, USA \\ $+1-817-272-0228$ \\ smiqbal@uta.edu
}

\begin{abstract}
Loosing motor activity due to impaired or damaged nerves or muscles affects millions of people world-wide. The resulting lack of mobility and/or impaired communication bears enormous personal, economical and social costs. While several assistive technologies exist, they rely on device surrogates to compensate for the lack of movement and thus provide limited utility and unnatural interface with the user. The ability of interfacing populations of neurons with super high-density multielectrode arrays (SD-MEA) can provide the sensing from and control of bionics devices by thought. Here we propose a neurointerfacing approach using SD-MEAs coated with carbon nanotubes and high-speed computing to overcome latency and long-term electrical viability bottlenecks that are essential in assistive environments. The proposed approach provides ability for fast integration of recording/stimulation from thousands of individually addressable electrodes, while coordinating a real-time computing approach to register, recognize, analyze and respond appropriately to the biological signals from the motor neurons and sensory signals from the robotic prosthesis.
\end{abstract}

\section{Categories and Subject Descriptors}

J.3 [Life and Medical Sciences]: High-speed data acquisition, analysis and decision making for neuronal stimulation.

\section{General Terms}

Measurement, Performance, Design, Reliability, Experimentation

\section{Keywords}

Carbon Nanotubes, Super High-Density Microelectrode Arrays (SD-MEA), Nanotechnology, Reliable Computing

\section{INTRODUCTION}

Interfacing the nervous system with microelectronics has benefited more than 110,000 hearing impaired adults and children with bionic cochlear implants. Recently, it has been demonstrated that voluntary movements of robotic prosthetic devices can be achieved with dexterity by extracting planned movement information from the motor cerebral cortex or the peripheral nerve

Permission to make digital or hard copies of all or part of this work for personal or classroom use is granted without fee provided that copies are not made or distributed for profit or commercial advantage and that copies bear this notice and the full citation on the first page. To copy otherwise, to republish, to post on servers or to redistribute to lists, requires prior specific permission and/or a fee.

PETRA'09, June 09-13, 2009, Corfu, Greece.

Copyright 2009 ACM ISBN 978-1-60558-409-6...\$5.00 via multi-electrode array interfaces $[1,2]$. Using this technology spinal cord injured patients can achieve computer cursor control and thus the ability to operate a robotic prosthetic device through imagined limb motions.

Sensory feedback can also be directly conveyed to amputees via electrical micro-stimulation of the sensory cortex [3] or peripheral nerves [4]. However, current neurointerfaces are quite limited for deployment in assistive environments owing to issues such as:

a. The control over the robotic limbs is short-lived and eventually fail due to immune reaction and tissue damage

b. Current approaches need high current injection for long-term stimulation, thus compromising the operational safety of these devices

c. A real-time computationally-reliable bidirectional close-loop neurointerface has yet to be developed

In this paper, we address the above issues by designing an innovative neurointerfacing approach that provides fast integration of recording/simulation from thousands of individually addressable electrodes sustained using high-speed real-time computing.

\section{ISSUES IN NEUROINTERFACES}

Most current neurointerfaces are designed as low density; hundred-microneedle electrode arrays for intact brain or nerve tissue penetration. The rigid electrode materials cause mechanical damage to the softer nerve tissue, due to both tissue micromotion and tethering forces imposed by the outside connectors. The resulting cellular damage can be massive and, in the cerebral cortex, obliterates entire neuronal columns around the electrode [5]. Thus the functionality is limited to short periods (weeks to months) and continued signal deterioration. Causes of such electrode failure include poor bio-abio interface, tissue damage by probe micromotion within the soft nerve tissue, and electrode insulation as a result of tissue scar formation [6, 7]. These limitations require neurointerface recalibration prior to each recording session and the gradual increase of the amount of electrical stimulation needed to maintain neural responses over time, which in turn raises the risk of electrolytic tissue damage and further compromises the long-term stability of the neurointerface. Thus, irrespective of the probe design, current electrode array sensors provide limited long-term functionality, both in brain- and nerve-machine interfaces [8]. Clearly, novel electrode interfaces are needed for long-term stability in sensitive recording and selective stimulation. Such a neurointerface must withstand continuous use through thousands of 
recording/stimulating cycles, without damaging the nerve tissue or compromising the neuron-electrode interface.

\section{HIGH-THROUHPUT APPROACH}

Metal microelectrodes have a typical impedance of $1 \mathrm{M} \Omega$ at body temperature $\left(37^{\circ} \mathrm{C}\right)$ at a bandwidth of $10 \mathrm{kHz}$. This equates to a thermal noise of almost $40 \mu \mathrm{V}$ peak-to-peak, not far from the approximately $100 \mu \mathrm{V}$ extracellular action potential signal level. The degree to which signals from a particular neuron can be discriminated from those originating from neighboring cells (the selectivity) is inversely proportional to the electrode surface area. However, the sensitivity of an electrode is maximized by minimizing the impedance of the electrode, which is attainable by decreasing surface area. This necessitates strategies to optimize the coupling and to balance the conflicting goals of a highly selective electrode with high sensitivity. Similarly, for stimulating the nerve, the coupling can be through direct transfer of electric current via a faradic process, or through capacitive charge transduction at an ionic double-layer. Gold, platinum, platinumiridium, tungsten, and tantalum are good candidates for faradic electrode-tissue contacts. However, capacitive electrodes such as titanium nitride reduce the risk of corrosion under stimulation conditions, and their performance varies highly with fabrication technology. Therefore, the capacitive reaction is inherently safer, but to ensure long-term viability of the neuron/electrode interface, it is desirable to minimize applied voltages and currents.

\subsection{Electrode Design with CNTs}

Carbon nanotubes (CNTs) are considered highly attractive for applications in nano electronics, field emission displays, and biochemical-sensors due to their excellent electrical and physical properties. Owing to their unique structure and electronic properties single-walled CNTs are extremely sensitive to the chemical environment [9]. The electronic properties of a given nanotube are dependent on the diameter, chirality as well as on applications. CNTs have great potential for continuous monitoring, diagnosis, and treatment of neural tissues as these can not only resist encapsulation of electrodes with undesirable glial scar tissue but also provide better electrical conductivity [10]. Recently we obtained evidence indicating that enticement of peripheral nerve regeneration through a non-obstructive multielectrode array (MEA), either after acute or chronic nerve amputation, offers a viable alternative to obtain early neural recordings and long-term interfacing of nerve activity (unpublished data).

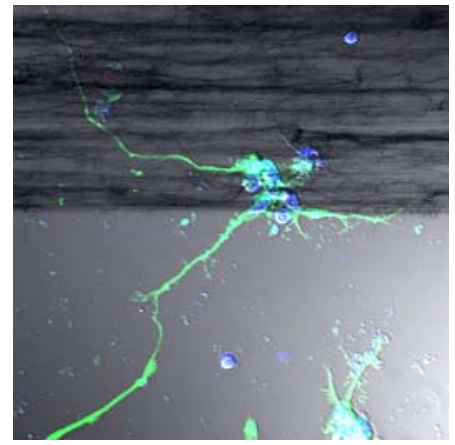

Figure 1. Cerebellar neurons stained with green phalloidin and counterstained with nuclear DAPI are shown growing on CNT (black-top half) and laminin coated substrate (bottom half)
We and others have also demonstrated that neurons grow onto pristine CNTs at rates comparable to the most permissive lamincoated substrates and that chemically modified CNTs offer a viable substrate for neuron recording and stimulation in vitro (Figure 1) [11-14]. Furthermore, CNT-gold or CNT-polypyrrole coating of platinum electrodes, enhances the electrical performance of the electrodes up to 45 fold by decreasing the impedance and increasing the sensitivity and charge storage capacity (Figure 2) [15].

Multichannel extracellular recording of action potentials from neuronal networks grown on MEAs is an effective methodology for analyzing the internal dynamics of spontaneously active nerve cells. The fabrication of MEAs incorporating CNT coatings bear the promise of providing enhanced bio/abio interface, increased recording sensitivity and reduced amount of current or voltage needed for neurostimulation, thus rendering a safer interface.
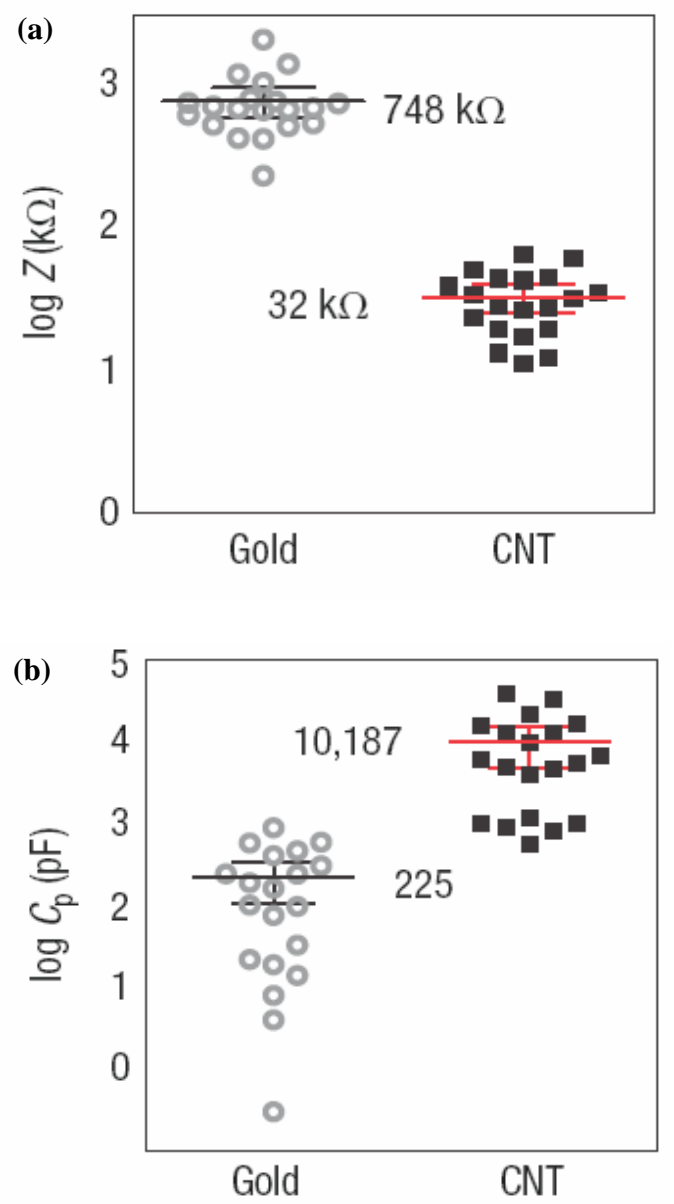

Figure 2. Gold and CNT composite coating of MEAs (a) reduced the impedance by 23 -folds, and (b) increased charge transfer 45-folds. Reprinted by permission from Macmillan Publishers Ltd: Nature Nanotechnology [15], copyright 2008.

\subsection{Advanced Asymmetric Core Processors and Real Time Neural Data Processing}

The analysis of extra-cellular neural recordings requires advanced computer systems and robust data acquisition electronics [16]. We propose using such systems to analyze and process the large 
neuro-sensor data in real-time. To this end, the data deluge created by measurements from hundreds and thousands of sensors precludes using traditional architectures. An alternative is to combine parallelism with specialization: investing a significant fraction of the hardware real estate to the acceleration of the critical compute kernels that dominate the execution time of most resource-demanding applications. The term "acceleration" refers to achieve a speedup that exceeds the number of cores used for the critical compute kernels. Amdahl's law suggests that the combined approach may prove more efficient than simply scaling out parallelism by replicating processor cores of current technology [17]. Moreover, multi-core processors with tightly coupled accelerators are becoming common, with the potential to sustain supercomputer-class node performance for dense computations, within a reasonable budget. Current products from major vendors package a few general-purpose cores (e.g., x86, PowerPC) and several accelerators (e.g., SIMD processors, GPUs), yielding power-efficient and low-cost compute nodes with performance exceeding 100 Gflops per chip [17-21]. Commoditization is now commonplace for asymmetric processors and systems with accelerators such as the Cell Broadband Engine (Cell) [22-24] in Sony Play Station 3 (PS3) (Astrophysicist Replaces Supercomputer with Eight PlayStation 3s; [24]), and NVIDIA GPUs [25, 26]. These processors are already driving several high-end computing platforms [27-32]. The Cell, in particular, was the first processor to enable sustained Petaflop performance in LANL's Roadrunner [27]. The use of off-the-shelf components in large-scale clusters is well established, both in academia, e.g., Condor [33], and industry, e.g., Google [34] and Amazon.com. Thus, it is natural that commoditization of accelerators will enable their use in analyzing large-scale data at a fraction of a budget of comparable traditional machines. However, using such devices for data processing in a real-time setting of thousands of MEAs recording action potentials is nontrivial requiring interactions between various Instruction Set Architectures and managing complex $\mathrm{I} / \mathrm{O}$ and data-intensive operations. Our current investigations have shown the use of specialized asymmetric core machines for data-intensive applications designing a data staging mechanism for Cell-based data processing $[35,36]$. Cell was used mainly because it was readily available at low cost in the Sony PS3, and it has been shown that by exploiting shared memory and asynchronous DMA operations the data processing could be sped up on Cell by as much as $24 \%$. Thus, it is clear that by careful orchestration of $\mathrm{I} / \mathrm{O}$ on asymmetric processors, the $\mathrm{I} / \mathrm{O}$ bottleneck can be removed even for massive data rates. This coupled with extreme compute densities of specialized cores such as those in Cells or GPUs, can provide support for real-time data processing, necessary for the neuro-sensor data analysis. This technology can allow real-time analysis of SD-MEA data and lay the foundation toward enabling real-world control of bionics in assistive environments.

\subsection{Super High Density MEA (SD-MEA)}

An ideal electrode in neuro-applications should be safe, biocompatible, highly sensitive, and offer stable coupling over long periods of time. To date, that ideal has not been attained. Our initial data shows recording/stimulating from dissociated cortical neurons seeded onto two, 32-electrode areas separated by $2 \mathrm{~cm}$. Electrical stimulation of cortical neurons seeded on each array and allowed to mature for 17-144 days before testing, revealed that neurons can be activated at lower stimulus voltages when grown on CNT-coated electrodes relative to uncoated ones, supporting the notion that that CNT sheets and perhaps CNTs more generally, can be used as stimulus interfaces for neuronal activation. Each electrode can detect the extracellular activity (action potentials) of several nearby neurons and can stimulate activity by passing a voltage or current through the electrode and across nearby cell membranes (e.g., $\pm 600 \mathrm{mV} 400 \mu \mathrm{s}$, biphasic pulses). Current work is focused on fabrication of SD-MEAs with thousands of sensing/stimulating electrodes for recording/stimulation of neurons in vitro and ultimately in vivo, and capabilities to

\section{a. Bias the sensing electrodes for stimulation and \\ b. Electrostatic capacitance measurement.}

The active biasing would (i) Have minimal effects from the ionic current flowing from the buffer species, and (ii) Be impervious to the tunneling currents between the electrodes (cross-talk). In our previous nano-electrode experiments in vacuum, we have observed tunneling currents in the range of $\mathrm{pA}$ between electrodes a few nanometers apart [37]. Very less tunneling is expected for our electrode design in the ionic solution. The capacitance of the electrodes before and after the neuron growth can be measured using capacitance/loss bridge. Change in capacitance can indicate change in the charge at constant voltage by $\Delta Q=\triangle C V(\triangle Q$ and $\triangle C$ are changes in charge and capacitance at constant voltage $V$ ). In the proposed electrode arrangement the current in the solution will be very well confined at the electrodes, thus the action potentials will be intercepted efficiently and result in modulation of the majority of the current flowing to the sensing electrodes.

The duty cycles for action potentials fall within fractions to a few milliseconds [38]. This necessitates measurements at rates of few $\mathrm{kHz}$. For measuring discrete voltage signals at these frequencies, with say 1000 electrodes at $1 \mathrm{kHz}$ duty cycle of measurement, more than a million data points need to be recorded faithfully in a second. Considering extracellular voltage signals to range between 100 to $500 \mu \mathrm{V}$, this would necessitate at least 800 discrete values at resolution of $0.5 \mu \mathrm{V}$, for each electrode. A lower limit can thus be approximated to 8 bits for one electrode (for $28=256$ discreet voltage levels). Given these considerations, the system has to be capable of a sustained measurement rate of at least 1 Megabyte/second. Given the large number of electrodes necessary for accurate and effective measurements $(10,000$ to 50,000 in $1 \mu^{2}$ ), and the need to adjust to faster rate of action potentials, we foresee the collective data rates to quickly become in excess of tens to hundreds of GB/s. Traditional approaches, with far less number of electrodes, have relied on dumping raw data to high-performance storage devices and processing it offline. Such an approach leads to long turn-around times between data collection and actual generation of useful electrical stimulants. For the assistive environments, such long delays are unacceptable. This gives a rationale for the need of computing techniques for real time neural data processing.

\section{OPPORTUNITY}

Sensitive, safe and sustainable neurointerfaces electrodes will benefit many applications, including the control of prosthetic limbs, neuro-prostheses, and Hybrid Bionic Systems, like exoskeletons and tele-operated platforms. Such computing techniques can also provide a solution to the data deluge faced in modern time-critical simulations and applications. A lot would be learned on how super-fast computing can find statistical patterns that can derive models, a new reality with huge datasets (pattern leading to models). Robust interface of electronic devices with electrogenic cellular systems can directly contribute towards computationally- 
reliable platforms for high-throughput screening of pharmaceutical or toxicological agents as well. Such interfaces can find integration with many technologies, e.g., wireless networks eliminating all external signal and power wiring.

\section{CONCLUSIONS}

This paper defines an approach of using SD-MEAs with CNTderived biocompatibility/electrical sensitivity and reliable highspeed real-time computing to sense and stimulate bionics devices in assistive environments. Such integration of cyber-physical systems can provide unmatched adaptability, autonomy, efficiency, functionality, reliability, and usability. High-speed data management coupled with rapid, faithful, stable and sensitive neurointerfaces can enhance human capabilities far beyond what is currently possible in assistive technologies.

\section{ACKNOWLEDGMENTS}

Authors acknowledge support from the Defense Advanced Research Projects Agency (MIR), the Crowely-Carter Foundation (MIR), NSF CAREER grant CCF 0746832 (ARB), and University of Texas at Arlington EE REP/RES grant (SMI).

\section{REFERENCES}

1. Hochberg, L.R., Serruya, M.D., Friehs, G.M., Mukand, J.A., Saleh, M., Caplan, A.H., Branner, A., Chen, D., Penn, R.D. and Donoghue, J.P. Neuronal ensemble control of prosthetic devices by a human with tetraplegia. Nature, 442(7099), 164-171, Jul 13, 2006.

2. Normann, R.A. Technology insight: future neuroprosthetic therapies for disorders of the nervous system. Nat Clin Pract Neurol, 3(8), 444-452, Aug, 2007.

3. Fitzsimmons, N.A., Drake, W., Hanson, T.L., Lebedev, M.A. and Nicolelis, M.A. Primate reaching cued by multichannel spatiotemporal cortical microstimulation. J Neurosci, 27(21), 5593-5602, May 23, 2007.

4. Dhillon, G.S. and Horch, K.W. Direct neural sensory feedback and control of a prosthetic arm. IEEE Trans Neural Syst Rehabil Eng, 13(4), 468-472, Dec, 2005.

5. Biran, R., Martin, D.C. and Tresco, P.A. Neuronal cell loss accompanies the brain tissue response to chronically implanted silicon microelectrode arrays. Exp Neurol, 195(1), 115-126, Sep, 2005.

6. Williams, J.C., Hippensteel, J.A., Dilgen, J., Shain, W. and Kipke, D.R. Complex impedance spectroscopy for monitoring tissue responses to inserted neural implants. $J$ Neural Eng, 4(4), 410-423, Dec, 2007.

7. Leung, B.K., Biran, R., Underwood, C.J. and Tresco, P.A. Characterization of microglial attachment and cytokine release on biomaterials of differing surface chemistry. Biomaterials, 29(23), 3289-3297, Aug, 2008.

8. Navarro, X., Krueger, T.B., Lago, N., Micera, S., Stieglitz, T. and Dario, P. A critical review of interfaces with the peripheral nervous system for the control of neuroprostheses and hybrid bionic systems J Peripher Nerv Syst, 2005, 229258.

9. Collins, P.G., Bradley, K., Ishigami, M. and Zettl, A. Extreme Oxygen Sensitivity of Electronic Properties of Carbon Nanotubes. Science, 287(5459), 1801, 2000.

10. Baughman, R.H., Zakhidov, A.A. and de Heer, W.A. Carbon nanotubes--the route toward applications. Science, 297(5582), 787-792, Aug 2, 2002.

11. Galvan-Garcia, P., Keefer, E.W., Yang, F., Zhang, M., Fang, S., Zakhidov, A.A., Baughman, R.H. and Romero, M.I.
Robust cell migration and neuronal growth on pristine carbon nanotube sheets and yarns. J Biomater Sci Polym Ed, 18(10), 1245-1261, 2007.

12. Mattson, M.P., Haddon, R.C. and Rao, A.M. Molecular functionalization of carbon nanotubes and use as substrates for neuronal growth. J Mol Neurosci, 14(3), 175-182, Jun, 2000.

13. Ni, Y., Hu, H., Malarkey, E.B., Zhao, B., Montana, V., Haddon, R.C. and Parpura, V. Chemically functionalized water soluble single-walled carbon nanotubes modulate neurite outgrowth. J Nanosci Nanotechnol, 5(10), 1707-1712, Oct, 2005.

14. Mazzatenta, A., Giugliano, M., Campidelli, S., Gambazzi, L., Businaro, L., Markram, H., Prato, M. and Ballerini, L. Interfacing neurons with carbon nanotubes: electrical signal transfer and synaptic stimulation in cultured brain circuits. $J$ Neurosci, 27(26), 6931-6936, Jun 27, 2007.

15. Keefer, E.W., Botterman, B.R., Romero, M.I., Rossi, A.F. and Gross, G.W. Carbon nanotube coating improves neuronal recordings. Nat Nanotechnol, 3(7), 434-439, Jul, 2008.

16. Wood, F. and Black, M.J. A nonparametric Bayesian alternative to spike sorting. J Neurosci Methods, 173(1), 1-12, Aug 15, 2008.

17. Hill, M.D. and Marty, M.R. Amdahl's Law in the Multicore Era. Computer, 41(7), 33-38, 2008.

18. Balakrishnan, S., Rajwar, R., Upton, M. and Lai, K., The Impact of Performance Asymmetry in Emerging Multicore Architectures in, IEEE Computer Society Washington, DC, USA, 506-517, 2005.

19. Pericas, M., Cristal, A., Cazorla, F.J., Gonzalez, R., Jimenez, D.A. and Valero, M., A Flexible Heterogeneous Multi-Core Architecture in, IEEE Computer Society Washington, DC, USA, 13-24, 2007.

20. Wong, H., Bracy, A., Schuchman, E., Aamodt, T.M., Collins, J.D., Wang, P.H., Chinya, G., Groen, A.K., Jiang, H. and Wang, H., Pangaea: a tightly-coupled IA32 heterogeneous chip multiprocessor in, ACM New York, NY, USA, 52-61, 2008.

21. AMD White Paper: The Industry-Changing Impact of Accelerated Computing, 2008.

22. Chen, T., Raghavan, R., Dale, J.N. and Iwata, E. Cell Broadband Engine Architecture and its first implementationA performance view. IBM Journal of Research and Development, 51(5), 559-572, 2007.

23. Kahle, J.A., Day, M.N., Hofstee, H.P., Johns, C.R., Maeurer, T.R. and Shippy, D. Introduction to the Cell multiprocessor. IBM Journal of Research and Development, 49(4/5), 589, 2005.

24. Corp, I. Cell Broadband Engine Architecture, 2007.

25. NC State Engineer Creates First Academic Playstation 3 Computing Cluster, March 8, 2007.

26. GraphStream, I. GraphStream scalable computing platform (SCP), 2006.

27. Barker, K.J., Davis, K., Hoisie, A., Kerbyson, D.J., Lang, M., Pakin, S. and Sancho, J.C., Entering the petaflop era: the architecture and performance of Roadrunner in, IEEE Press Piscataway, NJ, USA, 2008.

28. Blagojevic, F., Stamatakis, A., Antonopoulos, C.D. and Nikolopoulos, D., RAxML-Cell: Parallel Phylogenetic Tree Inference on the Cell Broadband Engine in 2007 IEEE International Parallel and Distributed Processing Symposium, 77, 2007. 
29. Gedik, B., Bordawekar, R.R. and Yu, P.S., CellSort: high performance sorting on the cell processor in Proceedings of the 33rd international conference on Very large data bases, VLDB Endowment, 1286-1297, 2007.

30. Héman, S. and Zukowski, M., Vectorized data processing on the cell broadband engine in Proceedings of the 3rd international workshop on Data management on new hardware, ACM New York, NY, USA, 2007.

31. Buehrer, G. and Parthasarathy, S. The Potential of the Cell Broadband Engine for Data Mining. Ohio State Univerity Technical Report OSU-CISRC-3/07--TR22, ftp://ftp. cse. ohio-state. edu/pub/tech-report/2007/TR22. pdf, 2007.

32. Bader, D.A. and Agarwal, V. FFTC: Fastest Fourier Transform for the IBM Cell Broadband Engine. LECTURE NOTES IN COMPUTER SCIENCE, 4873, 172, 2007.

33. Thain, D., Basney, J., Son, S.C. and Livny, M., The Kangaroo Approach to Data Movement on the Grid in Proceedings of the Tenth IEEE Symposium on High Performance Distributed Computing (HPDC10), 7-9, 2001.

34. Barroso, L.A., Dean, J. and Hölzle, U. Web Search for a Planet: The Google Cluster Architecture. IEEE MICRO, 2228, 2003.

35. Rafique, M.M., Butt, A.R. and Nikolopoulos, D.S., Dmabased prefetching for i/o-intensive workloads on the cell architecture in Proceedings of the 2008 conference on Computing frontiers, ACM New York, NY, USA, 23-32, 2008.

36. Rafique, M., Rose, B., Butt, A.R. and Nikolopoulos, D.S., CellMR: A Framework for Supporting MapReduce on Asymmetric Cell-Based Clusters in Proc. of the 23rd IEEE International Parallel and Distributed Processing Symposium (IPDPS), Rome, Italy, 2009.

37. Iqbal, S.M., Balasundaram, G., Ghosh, S., Bergstrom, D.E. and Bashir, R. Direct current electrical characterization of ds-DNA in nanogap junctions. Applied Physics Letters, 86(15), 153901, 2005.

38. Lewicki, M.S. A review of methods for spike sorting: the detection and classification of neural action potentials. Network: Computation in Neural Systems, 9(4), 53-78, 1998. 